\title{
Supersonic Flutter Utilization for Effective Energy-Harvesting Based on Piezoelectric Switching Control
}

\author{
Kanjuro Makihara ${ }^{1}$ and Shigeru Shimose ${ }^{2}$ \\ ${ }^{1}$ Department of Aerospace Engineering, Tohoku University, 6-6-01 Aramaki-Aza-Aoba, Aoba-ward, Sendai 980-8579, Japan \\ ${ }^{2} J a p a n$ Aerospace Exploration Agency (JAXA), Institute of Space and Astronautical Science (ISAS), 3-1-1 Yoshinodai, \\ Chuo-ward, Sagamihara, Kanagawa 252-5210, Japan
}

Correspondence should be addressed to Kanjuro Makihara, makihara@ssl.mech.tohoku.ac.jp

Received 4 January 2012; Revised 9 March 2012; Accepted 10 March 2012

Academic Editor: Osama J. Aldraihem

Copyright (c) 2012 K. Makihara and S. Shimose. This is an open access article distributed under the Creative Commons Attribution License, which permits unrestricted use, distribution, and reproduction in any medium, provided the original work is properly cited.

The harvesting of electrical energy generated from the flutter phenomenon of a plate wing is studied using the quasi-steady aerodynamic theory and the finite element method. The example of supersonic flutter structure comes from sounding rockets' wings. Electrical energy is harvested from supersonic flutter by using piezoelectric patches and switching devices. In order to evaluate the harvesting performance, we simulate flutter dynamics of the plate wing to which piezoelectric patches are attached. We demonstrate that our harvesting system can generate much more electrical energy from wing flutter than conventional harvesting systems can. This flutter utilization changes our perception to a useful one in various fruitful applications from a destructive phenomenon.

\section{Introduction}

Flutter is caused by the interaction between the structural motion of a wing and the aerodynamic load exerted on the wing. It is a typical self-excited aeroelastic phenomenon that occurs in wings, thin walls, and so on. Dowell [1] occurs most frequently within a high-speed, that is, transonic, supersonic, and hypersonic flow. Lottati [2] investigated the effects of structural and aerodynamic damping on the speed of flutter of a composite plate wing. Tang and Dowell [3] have analyzed the nonlinear behavior of a flexible rotor blade due to structural free-play and aerodynamic stall nonlinearities. The analytical results were compared with experimental observations. Various studies have been conducted on flutter dynamics, such as prediction of flutter and robust structural optimization of wings [4]. The use of sophisticated smart materials such as piezoelectric materials, shape memory alloys, and magnetostrictive materials in aerospace engineering can lead to the development of new design concepts. A new design concept is to alter structural dynamics by exertion of force or deformation. Moon and Hwang [5] used the linear quadratic regulator theory to suppress nonlinear panel flutter. Han et al. [6] designed a mu-synthesis controller to enhance flutter suppression performance despite parametric uncertainties. Raja et al. [7] used multilayer piezoelectric actuators and piezoelectric sensors for constructing a linear quadratic Gaussian controller to suppress the flutter of a composite plate. Agneni et al. [8] applied this passive method to flutter suppression and demonstrated satisfactory suppression performance. However, flutter suppression performance achieved by adopting this passive method is poorer when the electrical resonance frequency is slightly different from the frequency of the structure. Hence, the passive method provides limited robustness against model errors and is unsuitable for systems whose structural frequencies can shift because of aerodynamic influence.

Energy-harvesting (power-generation or power-scavenging) is a process by which energy is extracted from various sources and stored for future use, such as solar energy, tidal energy, piezoelectricity, thermoelectricity, and kinematic energy $[9,10]$. Energy-harvesting techniques are expected to be of vital importance in the future when fossil fuel reserves will exhaust completely. Among the various sources mentioned above, this study focuses on harvesting energy 
from vibrating structures using piezoelectricity. Cornwell et al. [11] developed an approach to improve energy output by using a tuned auxiliary structure.

Owing to the interest in the use of wind in harvesting energy, various studies and investigations were conducted by researchers. Robbins et al. [12] discussed vortex-induced oscillations of piezoelectric cantilevers located behind bluff bodies. Kwon [13] conducted an investigation of a simple Tshaped cantilever design with a mass flow controller (MFC) at a low air flow speed.

Thus far, wing flutter has been considered as a phenomenon that should be avoided, especially in aerospace engineering. However, adding electromotor or piezoelectric transducers to wings under aeroelastic vibrations is a feasible method for harvesting energy, as Isogai et al. [14] verified in simulation and experiments. This research used the conventional rectified harvester composed of a diodebridge. Bryant and Garcia [15] proposed a piezoelectric harvester using a conventional rectified harvester based on the impedance matching method. De Marqui et al. [16, 17] proposed an electromechanically coupled finite element model that was combined with an unsteady aerodynamic model. They also developed a piezoelastic model for air flow excitation of cantilevered plates using doublet-lattice method. Dunnmon et al. [18] presented an aeroelastic energy harvester that was exploited for piezoelectric power generation from aerodynamic flows, especially nonlinear limit cycle oscillations. Sousa et al. [19] presented modeling and experiments of aeroelastic energy harvesting using piezoelectric transduction with a focus on exploiting combined nonlinearities. Erturk et al. [20] investigated the concept of piezoaeroelasticity for energy harvesting and focused on mathematical modeling and experimental validations of the problem of generating electricity at the flutter boundary of a piezoaeroelastic airfoil. Although these previous studies showed the possibility of energy-harvesting from flutter phenomenon, they used only a simple rectified harvester and their flutter performances were not high. Therefore, in order to enhance the flutter harvesting performance, we introduce an energy-harvesting system that extracts electrical energy from wing flutter using a switching control with a piezoelectric material. Furthermore, we modified the switching control to adopt to flutter systems that are subject to the change in natural frequency. This paper clearly shows the difference between the conventional flutter harvester and our new harvester, by explaining both energy-harvesting mechanisms in detail. The effective use of wing flutter changes our perception of it-from being a destructive phenomenon as it was formerly known to be to being useful in various applications.

\section{Aerodynamic Pressure of Tail Wing of Sounding Rocket}

2.1. Sounding Rocket. In this study, we focus on harvesting energy from the wing flutter of a sounding rocket [21]. Institute of Space and Astronautical Science (ISAS), Japan Aerospace Exploration Agency (JAXA), has developed

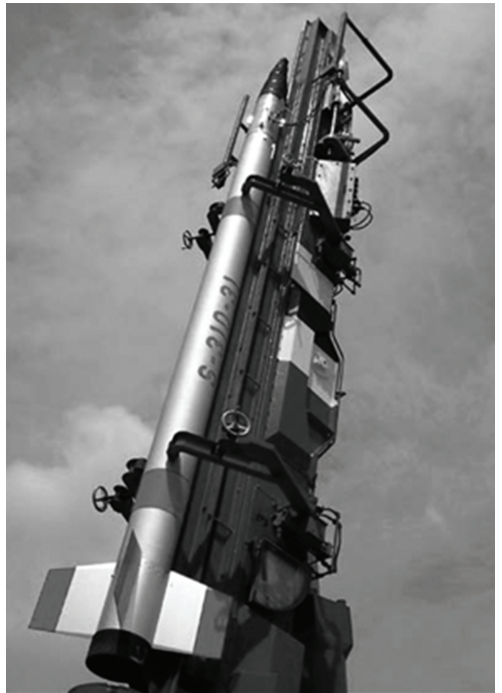

FIgURE 1: Sounding rocket having its four titanium tail wings.

a sounding rocket S-310 (Figure 1), which we consider as an example in our study. The outline of this rocket is explained in brief here to highlight its characteristics. The S-310 is a single-stage rocket, $310 \mathrm{~mm}$ in diameter; it can reach an altitude of $150 \mathrm{~km}$. The rocket attains approximately Mach 4.6 in 23 seconds, which indicates that it flies at a supersonic speed during most of its flight. The sounding rocket has four tail wings (fins) made of a solid titanium plate.

2.2. Aerodynamic Pressure of Tail Wing for Flutter Interaction. A titanium plate simulates the wings of a sounding rocket flying at a supersonic speed, as Figure 2 depicts. Thus, the plate can be subject to supersonic flutter during the flight of the sounding rocket. We include a cantilevered-plate wing in the study to investigate the harvesting of energy from wing flutter. Piezoelectric patches are attached to the wing surface to generate electrical energy from the motion of the wing. In reality, patches attached to the wing surface may adversely affect fluid dynamics because they cause discontinuity of the wing surface. Furthermore, the patches may be adversely affected by the heat generated by aerodynamic interference. However, in this study, we do not consider these issues. Nevertheless, we need to develop piezoelectric actuators such as piezoelectric fibers embedded in composite plate wings.

Aerodynamic pressure at a high supersonic speed is described by a quasi-steady first-order piston theory [22]. The pressure exerted on the wing plate of a rocket flying at a speed $U$ is given by

$$
p-p_{\infty}=\frac{\rho_{\infty} U^{2}}{\sqrt{M^{2}-1}}\left[\frac{\partial u}{\partial y}+\frac{M^{2}-2}{M^{2}-1} \frac{1}{U} \frac{\partial u}{\partial t}\right]
$$

where

$$
M \equiv \frac{U}{a_{\infty}} .
$$




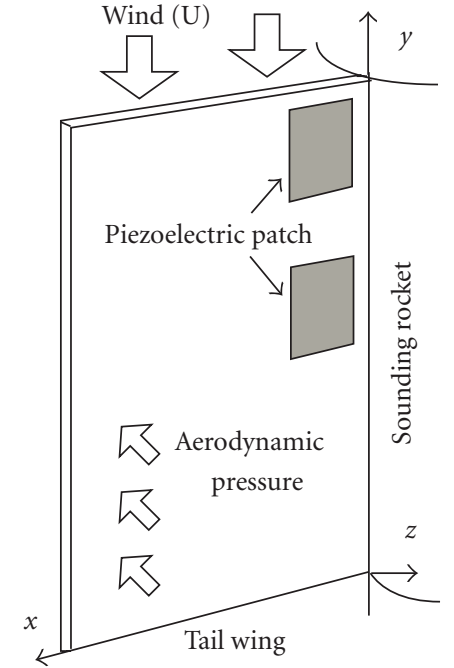

FIgURE 2: Tail wing subject to flutter and piezoelectric patches attached to the wing.

Assuming that the pressure is exerted on both sides of the wing, we can express the resultant pressure, $p_{a}(x, y, t)$, exerted on the wing as

$$
p_{a}(x, y, t) \equiv \frac{-2 \rho_{\infty} U^{2}}{\sqrt{M^{2}-1}}\left[\frac{\partial u}{\partial y}+\frac{M^{2}-2}{M^{2}-1} \frac{1}{U} \frac{\partial u}{\partial t}\right] .
$$

\section{Harvesting Scheme for Wing with Piezoelectric Patches}

3.1. Equation of Motion with Aerodynamic. Piezoelectric patches shown in Figure 2 are assumed to be polarized in the thickness direction ( $z$-direction) and isotropic in the in-plane direction $(x-y$ plane). Hence, their constitutive equations [23] are given by

$$
\sigma_{p}=\mathbf{c}_{p}^{D} \varepsilon_{p}-\mathbf{h} D_{z}, \quad E_{z}=-\mathbf{h}^{T} \varepsilon_{p}+\beta_{z z}^{S} D_{z},
$$

where

$$
\begin{gathered}
\sigma_{p} \equiv\left\{\begin{array}{c}
\sigma_{x} \\
\sigma_{y} \\
\tau_{x y}
\end{array}\right\}, \quad \epsilon_{p} \equiv\left\{\begin{array}{c}
\epsilon_{x} \\
\epsilon_{y} \\
\gamma_{x y}
\end{array}\right\}, \quad \mathbf{h} \equiv\left\{\begin{array}{c}
h_{x z} \\
h_{x z} \\
0
\end{array}\right\}, \\
\mathbf{c}_{p}^{D} \equiv \frac{c_{p}^{D}}{1-v_{p}^{2}}\left[\begin{array}{ccc}
1 & v_{p} & 0 \\
v_{p} & 1 & 0 \\
0 & 0 & \frac{1-v_{p}}{2}
\end{array}\right] .
\end{gathered}
$$

The stress-strain relation of a wing is written as

$$
\sigma_{w}=\mathbf{c}_{w} \varepsilon_{w},
$$

where

$$
\mathbf{c}_{w} \equiv \frac{c_{w}}{1-v_{w}^{2}}\left[\begin{array}{ccc}
1 & v_{w} & 0 \\
\nu_{w} & 1 & 0 \\
0 & 0 & \frac{1-v_{w}}{2}
\end{array}\right] .
$$

The linear strain-displacement relation based on the Kirchhoff-Love assumption is

$$
\varepsilon=-\mathrm{z}\left[\frac{\partial^{2}}{\partial \mathrm{x}^{2}}, \frac{\partial^{2}}{\partial \mathrm{y}^{2}}, 2 \frac{\partial^{2}}{\partial \mathrm{x} \partial \mathrm{y}}\right]^{T} u(x, y, t) .
$$

On the surface of the wing, $n_{p}$ pieces of piezoelectric patches are attached, and the $j$ th patch $\left(1 \leq j \leq n_{p}\right)$ is attached at positions $x_{1 j} \leq x \leq x_{2 j}, y_{1 j} \leq y \leq y_{2 j}$, and $z_{1 j} \leq z \leq$ $z_{2 j}$. To ensure the generality of this theoretical analysis, a multiple-input-multiple-output system is considered. Using Hamilton's principle, we can construct

$$
\int_{t_{1}}^{t_{2}}\left[\delta T_{w}-\delta U_{w}+\sum_{j=1}^{n_{p}}\left(\delta T_{p j}-\delta U_{p j}\right)+\delta W\right] \mathrm{d} t=0
$$

where

$$
\begin{aligned}
& T_{w} \equiv \int_{V} \frac{1}{2} \rho_{w}\left(\frac{\partial u}{\partial t}\right)^{2} \mathrm{~d} V, \quad U_{w} \equiv \frac{1}{2} \int_{V} \sigma_{w}^{\mathrm{T}} \epsilon_{w} \mathrm{~d} V \\
& T_{p j} \equiv \int_{V_{p j}} \frac{1}{2} \rho_{p j}\left(\frac{\partial u}{\partial t}\right)^{2} g_{j}(x, y, z) \mathrm{d} V \\
& U_{p j} \equiv \frac{1}{2} \int_{V_{p j}}\left(\sigma_{p}^{\mathrm{T}} \epsilon_{p}+E_{z} D_{z}\right) \mathrm{d} V \\
& g_{j}(x, y, z) \equiv {\left[H\left(x-x_{1 j}\right)-H\left(x-x_{2 j}\right)\right] } \\
& \times\left[H\left(y-y_{1 j}\right)-H\left(y-y_{2 j}\right)\right] \\
& \times\left[H\left(z-z_{1 j}\right)-H\left(z-z_{2 j}\right)\right] .
\end{aligned}
$$

Virtual work, $\delta W$, can be written as

$$
\begin{aligned}
\delta W \equiv & \int_{S} \delta u\left[f(x, y, t)+p_{a}(x, y, t)\right] \mathrm{d} S \\
& +\sum_{j=1}^{n_{p}} V_{j} \int_{S_{p j}} \delta D_{z j} g_{j}(x, y, z) \mathrm{d} S .
\end{aligned}
$$

Here, $f(x, y, t)$ is the external force normal to the wing and $V_{j}$ is the voltage applied to the $j$ th piezoelectric patch as a generalized external force.

The finite element method (FEM) element proposed by Zienkiewicz and Taylor (known as the ACM element) [24], which is a four-node nonconforming plate element, is employed to discretize the partial derivative equations of motion. From (3)-(11), the equation of motion for the cantilevered wing with multiple piezoelectric patches attached to it can be expressed as

$$
\mathbf{M} \ddot{\mathbf{x}}+\mathbf{K x}+\mu \mathbf{A x}=\mathbf{B Q}+\mathbf{f},
$$

where

$$
\begin{gathered}
\mathbf{M} \equiv \sum_{\text {ele }} \int_{V+V_{p}} \rho \mathbf{N}_{F}^{T} \mathbf{N}_{F} \mathrm{~d} V, \quad \mathbf{K} \equiv \sum_{\text {ele }} \int_{V+V_{p}} z^{2} \mathbf{B}_{F}^{T} \tilde{\mathbf{c}}_{F} \mathrm{~d} V, \\
\mathbf{A} \equiv \sum_{\text {ele }} \int_{S} \mathbf{N}_{F}^{T} \frac{\partial \mathbf{N}_{F}}{\partial y} \mathrm{~d} S, \quad \mathbf{B} \equiv \sum_{\text {ele }} \int_{V_{p}}\left(\frac{z}{S_{\text {ele }}}\right) \mathbf{B}_{F}^{T} \mathbf{h} d V, \\
\mathbf{B}_{F} \equiv\left[\frac{\partial^{2} \mathbf{N}_{F}}{\partial x^{2}}, \frac{\partial^{2} \mathbf{N}_{F}}{\partial y^{2}}, 2 \frac{\partial^{2} \mathbf{N}_{F}}{\partial x \partial y}\right]^{T}, \quad \mu \equiv \frac{2 \rho_{\infty} U^{2}}{\sqrt{M^{2}-1}} .
\end{gathered}
$$


A vector-matrix form equation for piezoelectric voltage is described as

$$
\mathbf{V}=-\mathbf{B}^{\mathrm{T}} \mathbf{x}+\mathbf{C}^{-1} \mathbf{Q},
$$

where

$$
\mathbf{C}^{-1} \equiv \sum_{\text {ele }} \int\left(\frac{z \beta_{z z}^{S}}{S_{\text {ele }}}\right) \mathrm{d} z
$$

We perform the transformation $\mathbf{x}=\boldsymbol{\Phi} \boldsymbol{\eta}$ and introduce the modal damping ratio in (12) as

$$
\ddot{\boldsymbol{\eta}}+\boldsymbol{\Xi} \dot{\boldsymbol{\eta}}+\left(\boldsymbol{\Omega}+\mu \boldsymbol{\Phi}^{T} \mathbf{A} \boldsymbol{\Phi}\right) \boldsymbol{\eta}=\boldsymbol{\Phi}^{T} \mathbf{B Q}+\boldsymbol{\Phi}^{T} \mathbf{f},
$$

where

$$
\begin{gathered}
\Phi \equiv\left[\phi_{1}, \phi_{2}, \ldots, \phi_{n}\right], \quad \Omega \equiv \operatorname{diagonal}\left[\omega_{k}^{2}\right], \\
\Xi \equiv \operatorname{diagonal}\left[2 \zeta \omega_{k}\right] .
\end{gathered}
$$

This eigenvalue problem is solved for the homogenous part of (12) without aerodynamics (i.e., $\mu=0$ ).

3.2. Piezoelectric Switching Control for Energy-Harvesting. A conventional energy-harvesting device that employs piezoelectric materials to generate energy includes a vibrating piezoelectric structure and an energy storage system $[9,10]$. The energy generator is composed of piezoelectric materials, which are attached to the structure, and a harvesting circuit. Circuit I shown in Figure 3 is a conventional harvesting circuit consisting of a diode bridge of four diodes. The diode bridge is connected to the piezoelectric materials and it provides a mechanism for current rectification. When the piezoelectric voltage $V_{p}$ is positive, electric current starts to flow in one loop (from A, B, D, F, C, E, and to G), and $V_{s}$ instantly becomes equal to $V_{p}$. When $V_{p}$ is negative, electric current starts to flow in one loop (from G, E, D, F, C, B, and to A), and $V_{s}$ instantly becomes equal to $-V_{p}$. This harvesting system is used in many vibration systems; however, it does not satisfactorily harvest electrical energy.

To enhance the energy-harvesting performance, we use the energy-recycling semiactive approach [25] as an effective energy-harvesting mechanism. This approach involves the use of Circuit II, which is connected to the piezoelectric material, as shown in Figure 4. The circuit has only two diodes and a selector switch. The selector switch is connected to point 1 or 2 to control the current flow. According to our previous study [25], the advanced energy-recycling approach can simultaneously manage multiple circuits and piezoelectric patches. The harvesting system is assumed to consist of circuits and $n_{p}$ pieces of piezoelectric patches. One switching strategy of managing vibration semiactively involves controlling the $j$ th switch $\left(1 \leq j \leq n_{p}\right)$ so that $Q_{j}$ has the same polarity as $Q_{T j}$ and the absolute value of $Q_{j}$ is maximum. Here, $Q_{T j}$ is the active feedback input that is determined by an active control scheme. The switching logic [25] is

when $Q_{T j}<0$, turn $j$ th switch to point 1, when $Q_{T j}>0$, turn $j$ th switch to point 2.

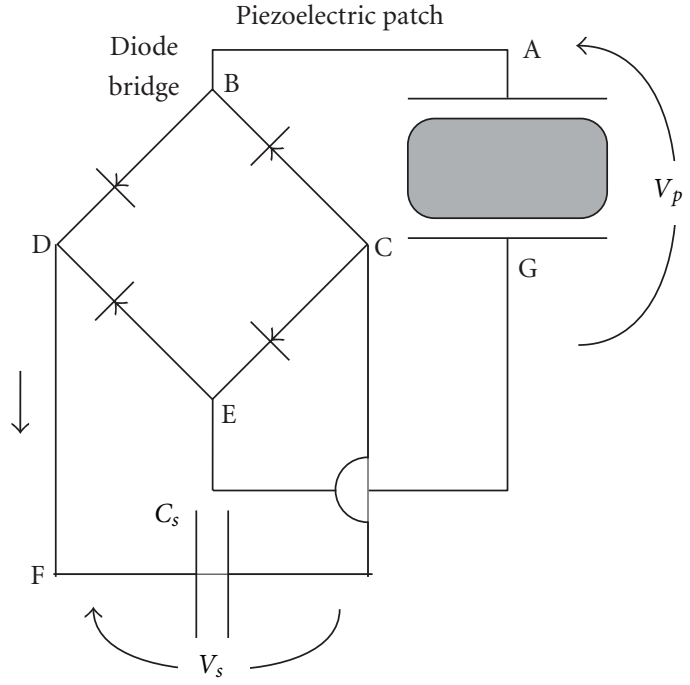

FIgURE 3: Circuit I-conventional harvesting circuit.

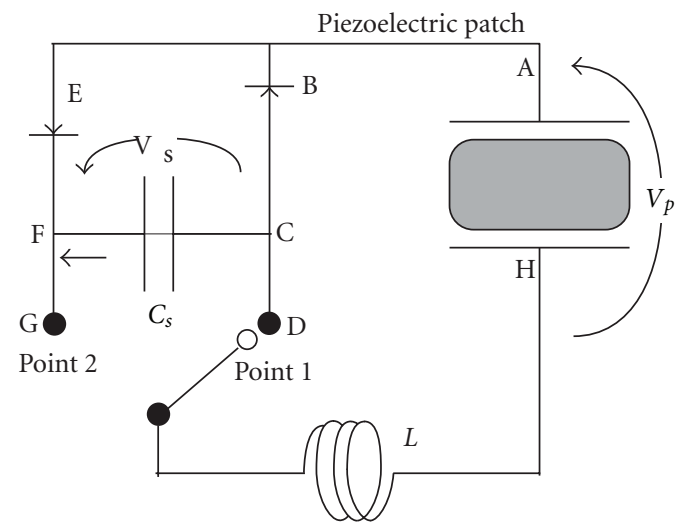

FIgURE 4: Circuit II-efficient harvesting circuit with energyrecycling mechanism.

Because our semiactive method just changes the switch connection to point 1 or 2 , it never increases the vibration energy by its switching action. Hence, we can infer that our semiactive approach is safer than other active approaches that usually involve a risk of instability such as spillover.

As will be seen later, in flutter problems, modal frequencies can shift according to dynamic pressure. Therefore, sophisticated controls based on modal decomposition are impractical. One way of implementing switching controls without modal information is to adopt an approach based on the direct velocity feedback method [26], that is, describing $Q_{T j}$ as

$$
Q_{T j} \equiv-\dot{\varepsilon}_{p j},
$$

where $\varepsilon_{p j}$ is the strain at the position of the $j$ th piezoelectric patch.

3.3. Energy-Harvesting Mechanism. This study focuses on the energy harvested from wing flutter via piezoelectric 


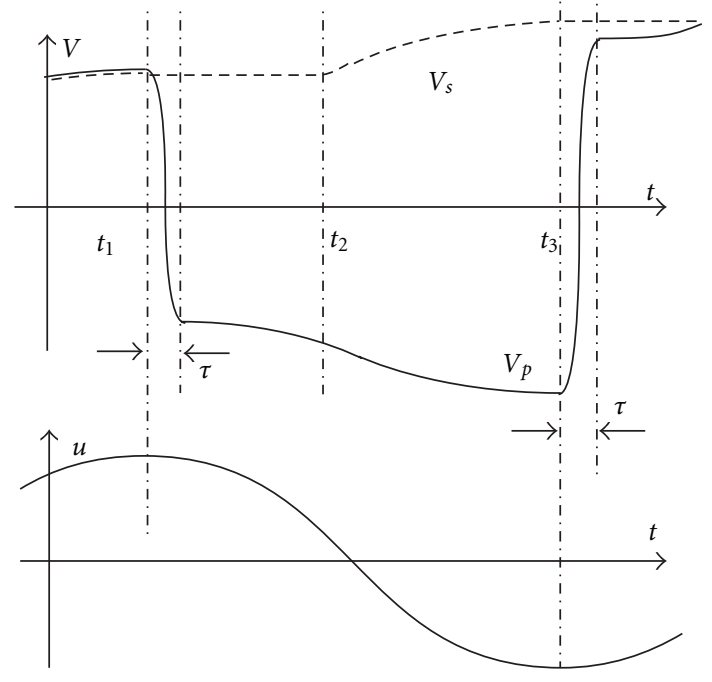

FIGURE 5: Illustration of energy-harvesting mechanism with switching control.

patches. We here explain the harvesting mechanism shown in Circuit II (Figure 4) using a single-degree-of-freedom (SDOF) system, as this system is comprehensible. Figure 5 shows a schematic of the single-mode vibration in energyharvesting. When the velocity $V_{p}$ is negative, the switch is connected to point 2, whereas when $V_{p}$ is positive, the switch is connected to point 1 . When the displacement reaches a peak and when $V_{p}$ is positive (e.g., $t=t_{1}$ in Figure 5), the polarity of $V_{p}$ is expected to change from positive to negative. When the selector switch is connected to point 1 from point 2, electric current starts to flow in one loop (from A, E, F, $\mathrm{G}, \mathrm{L}$, and to $\mathrm{H}$ ). Because of this current flow, the polarity of $V_{p}$ reverses to negative. After $V_{p}$ reaches the minimum peak, the diode between points $\mathrm{E}$ and $\mathrm{F}$ prevents electric current from flowing in the opposite direction, and $V_{p}$ retains the minimum negative value. This current flow is responsible for the voltage reversal mechanism. After the completion of this process and while $-V_{p}$ is lesser than $V_{s}$, no electric current flows in any branch circuit. During this time, $V_{p}$ decreases according to the structural motion because of the effect of piezoelectric materials. As soon as $-V_{p}$ reaches $V_{s}$ (e.g., $t=t_{2}$ in Figure 5), electric current starts to flow in one loop (from H, L, G, F, C, B, and to A), and some electric charge is stored in the capacitor. This current flow is responsible for the energy-harvesting mechanism.

To summarize the above discussion, once the connection point of the selector switch is changed, the voltage-reversal and energy-harvesting processes automatically proceed in the circuit.

\section{Numerical Simulation}

We carried out a numerical simulation of the energy-harvesting on the plate wing (Figure 2). The wing had an area of $0.37 \times 0.49 \mathrm{~m}$ and a thickness of $6.75 \mathrm{~mm}$. It was made of a titanium alloy (Ti-6Al-4V). This configuration is essentially a simpler model of the S-310's tail wing for explaining the
TABle 1: Parameters of titanium wing and piezoelectric patch.

\begin{tabular}{lccc}
\hline & Unit & $\begin{array}{c}\text { Piezoelectric } \\
\text { patch }\end{array}$ & $\begin{array}{c}\text { Titanium } \\
\text { wing }\end{array}$ \\
\hline Piezoelectric coefficient & $10^{8} \mathrm{~V} / \mathrm{m}$ & 4.67 & $\mathrm{NA}$ \\
Dielectric coefficient & $10^{7} \mathrm{Vm} / \mathrm{C}$ & 1.95 & $\mathrm{NA}$ \\
Density & $10^{3} \mathrm{~kg} / \mathrm{m}^{3}$ & 8.10 & 4.47 \\
Young's modulus & $10^{10} \mathrm{~N} / \mathrm{m}^{2}$ & 6.40 & 11.3 \\
Poisson's ratio & $\mathrm{NA}$ & 0.32 & 0.31 \\
\hline
\end{tabular}

harvesting performance on which we focus in this study. The wing was rigidly supported on one of its boundaries, that is, $x=0$. A piezoelectric patch (ceramic type, $154 \times$ $175 \times 0.5 \mathrm{~mm}$ ) was attached at $0 \leq x \leq 0.154$ and $0.28 \leq$ $y \leq 0.455$ on the wing. The total resistance in the circuit was $30 \Omega$, and the piezoelectric capacitance $C_{p}$ was $1.17 \times$ $10^{-6} \mathrm{~F}$. Without considering the aerodynamic influence, the first and second mode frequencies of the open circuit (i.e., constant charge) were $43.0 \mathrm{~Hz}$ and $84.5 \mathrm{~Hz}$, respectively. The simulation parameters are listed in Table 1 . These parameters were determined on the basis of the materials used in the investigation to carry out realistic simulations.

4.1. Eigenvalue Analysis of Flutter. Equation (12) can be reduced into an eigenvalue problem:

$$
\operatorname{det}[-\lambda \mathbf{M}+\mathbf{K}+\mu \mathbf{A}]=0,
$$

where $\lambda$ is a complex eigenvalue. Because the eigen analysis is performed on a no-control system, the control input and the external disturbance are neglected. Figure 6 plots eigenvalues as a function of the dynamic pressure parameter $\mu$. The curves of the two values of the real part approach each other as $\mu$ increases. When $\mu=2.86 \times 10^{6}$, the two values of the real part can be combined as $\operatorname{Re}[\lambda]=1.86 \times 10^{3}$. At this critical value of $\mu$ the wing experiences a flutter phenomenon. This figure shows the loci of only the first and second modes. Since this critical value indicates the smallest dynamic pressure among all critical values, we focus on the relation between only two vibration modes.

4.2. Simulations of Harvesting Performance. We simulated flutter dynamics in the case that a white noise force was exerted on the wing surface. The power spectral density (PSD) per unit frequency of the random force (white noise) had a constant value of $0.1 \mathrm{~N}^{2} / \mathrm{Hz}$ in the range of 30 to $100 \mathrm{~Hz}$ and a value of 0 in the rest of the frequency range. Therefore, the frequency range of the nonzero PSD covered the first and second modes.

The history of wing dynamics at a critical dynamic pressure (i.e., $\mu=2.86 \times 10^{6}$ ) is shown in Figure 7. This history shows the dynamics in the case of the switching control with Circuit II for the purpose of energy-harvesting. Piezoelectric voltage, storage voltage, electric charge, tip displacement at the wing corner, and input random force are shown. The tip displacement increases due to the flutter phenomenon, and accordingly, both voltages and charge also increase. The storage capacitor has a storage capacitance, 


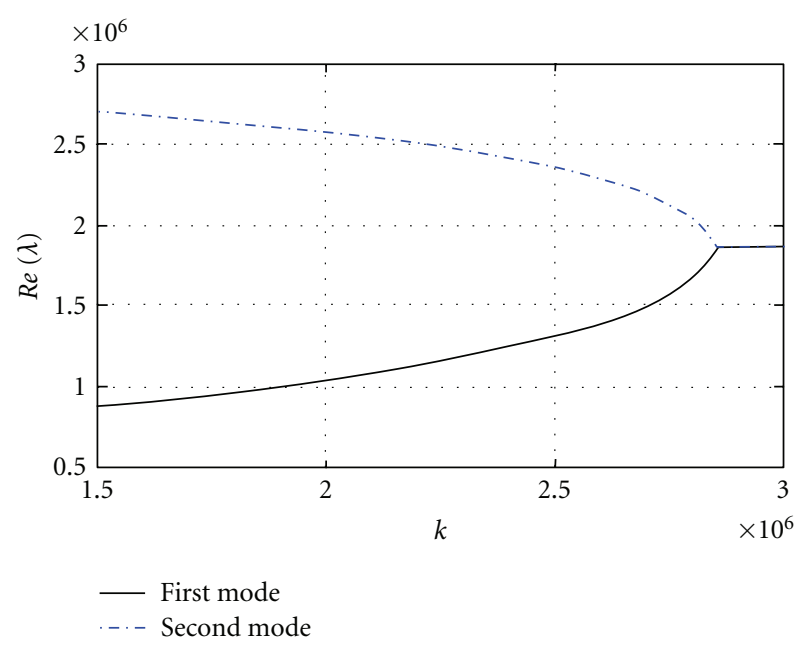

(a)

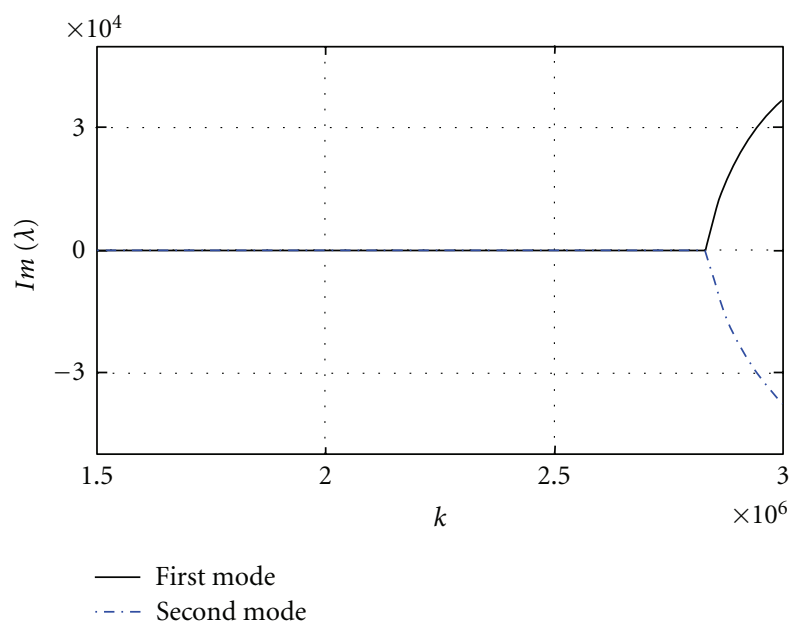

(b)

Figure 6: Eigenvalues as a function of dynamic pressure.

$C_{s}$, of $1.17 \times 10^{-6} \mathrm{~F}$. At $t=0.2 \mathrm{~s}$, the tip displacement reached $4.1 \times 10^{-3} \mathrm{~m}$. Because of the piezoelectric effect at this point, the wing experienced a flutter phenomenon; the corresponding piezoelectric voltage was $79.1 \mathrm{~V}$.

Figure 8 shows history of flutter harvesting with Circuit II for a zoomed time scale of Figure 7. Due to the energyrecycling mechanism, the piezoelectric voltage alternatively takes positive and negative values. The relation between piezoelectric and stored voltages is clearly depicted. The stored voltage increases only if the absolute value of piezoelectric voltage reaches the value of stored voltage, and, after then, both are equal. This equality indicates that the electrical energy is transferred to the stored capacitor. We can confirm that electric charge is constant while energy is not transferred, whereas the amount of electric charge decreases while energy is transferred. These behaviors of both voltages agree with the explanation of energy-harvesting in Figure 5.

Figure 9 shows history of flutter harvesting with Circuit I for a zoomed time scale. The piezoelectric voltage is quite different from that of history of energy-harvester with Circuit II (Figure 8), and is a sine wave based on the piezoelectric effect of structural vibration. Compared with Figures 8 and 9, the energy-recycling mechanism enables the stored voltage to be larger, which means that Circuit II is much more effective for flutter-harvester than the conventional system.

Further, the storage capacitor and diode bridge were connected to the harvesting circuit. The storage capacitance is expressed with a capacitance ratio as $\kappa \equiv C_{s} / C_{p}$. Figure 10 shows the voltage in the two harvesting systems with Circuit I and Circuit II for $\kappa=1.0$. The line for the harvesting system with Circuit I indicates the conventional method, whereas that with Circuit II indicates the new harvesting method. Clearly, the harvesting system with Circuit II performs better in harvesting energy from wing flutter. The voltage in the harvesting device, $V_{s}$, is shown to have a step-like curve, which is characteristic of harvesting systems that use diode bridges. Stored electrical energy can be described as
$(1 / 2) C_{s} V_{s}^{2}$. Our harvesting system with Circuit II generates $6.7 \times 10^{-2} \mathrm{~J}$ at $t=0.25 \mathrm{~s}$, whereas the system with Circuit I generates $5.2 \times 10^{-3} \mathrm{~J}$. Therefore, our flutter harvesting system can generate 10 times more electrical energy than the conventional system. The generation ratio, that is, 10 times, is quite a striking and attractive number as a powerful energy harvester. Our harvesting system shows potential and is effective because it takes advantage of the wing flutter that has thus far been considered as destructive.

To extensively assess the harvesting performance, we carried out further simulations with various values of storage capacitance $C_{s}$. The time-averaged electrical power was calculated with each capacitance ratio $\kappa$ between $0 \mathrm{~s}$ and $0.3 \mathrm{~s}$. Figure 11 shows the electrical power as a function of $\kappa$. Interestingly, the harvesting system with Circuit I harvested the maximum power with approximately $\kappa=31.6$, whereas the system with Circuit II harvested the maximum power with approximately $\kappa=5.62$. The result indicates that the optimum storage capacitance that harvests the maximum power should be incorporated into the harvesting system.

\section{Discussion on Supersonic Flutter Utilization for Energy-Harvesting}

This section contains a discussion on the utilization of supersonic flutter for energy-harvesting.

Firstly, so far we have looked at rocket wings as an example feasibility study of harvesting the energy produced by supersonic flutter. However, any structure, as long as the flutter phenomenon occurs, can be used for flutter harvesting. Possible examples are harvesting flutter within thin flag-type structures using flexible piezoelectric film, and harvesting panel flutter in fuselages using ceramictype piezoelectric patches, in addition to the wing flutter discussed here. 


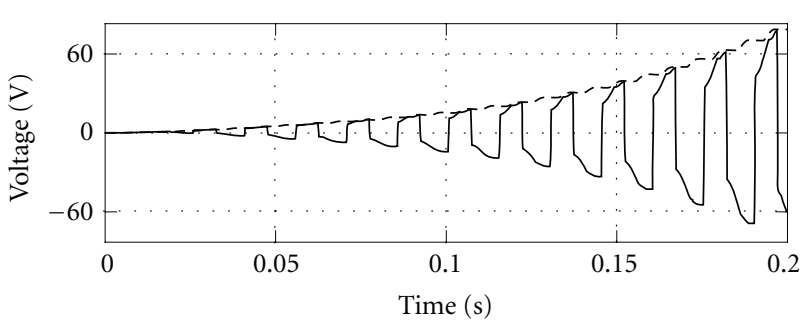

(a)

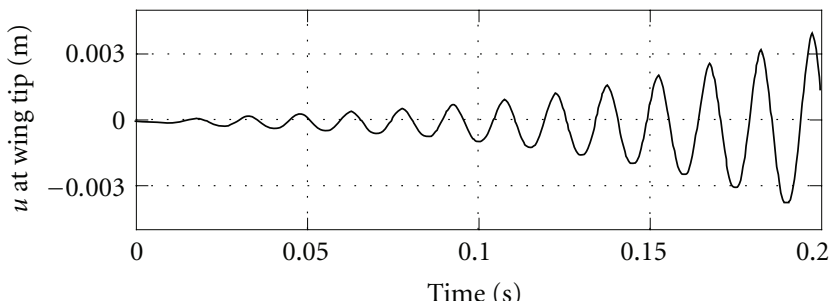

— Piezoelectric voltage

- - - Storage voltage

(c)

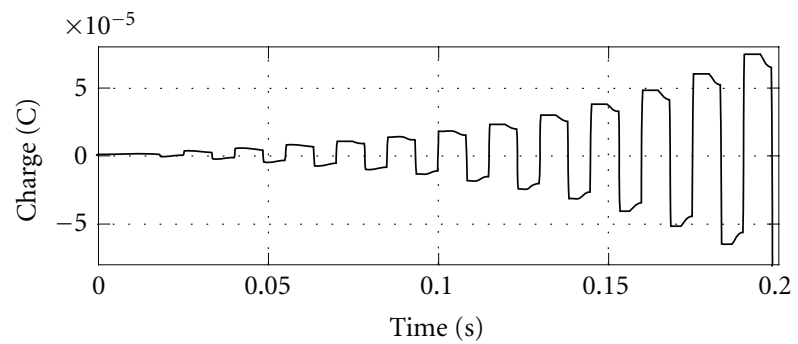

(b)

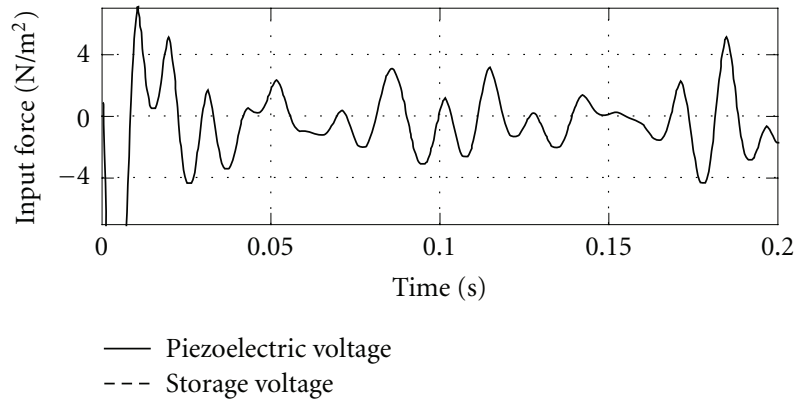

(d)

FIGURE 7: Timeline of the tail wing at critical dynamic pressure with energy-harvesting (Circuit II).

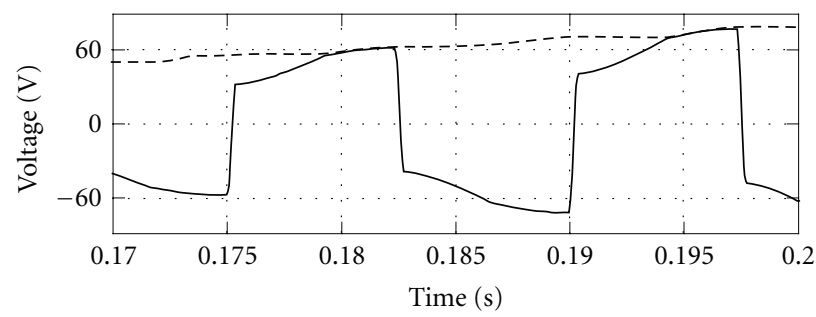

- Piezoelectric voltage

- - - Storage voltage

(a)

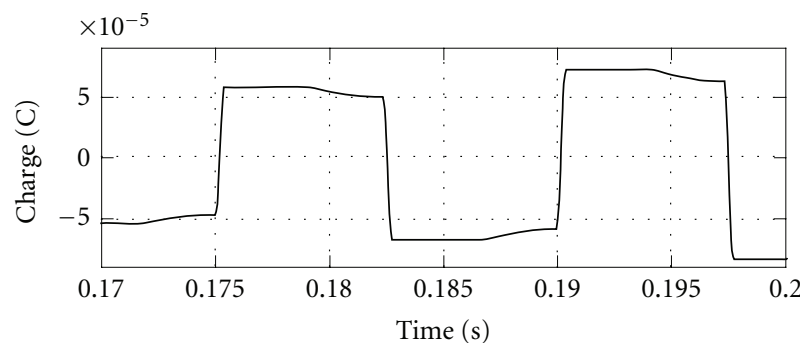

- Piezoelectric voltage

- - Storage voltage

Figure 8: Magnified view of timeline of the tail wing with energy-harvesting (Circuit II).

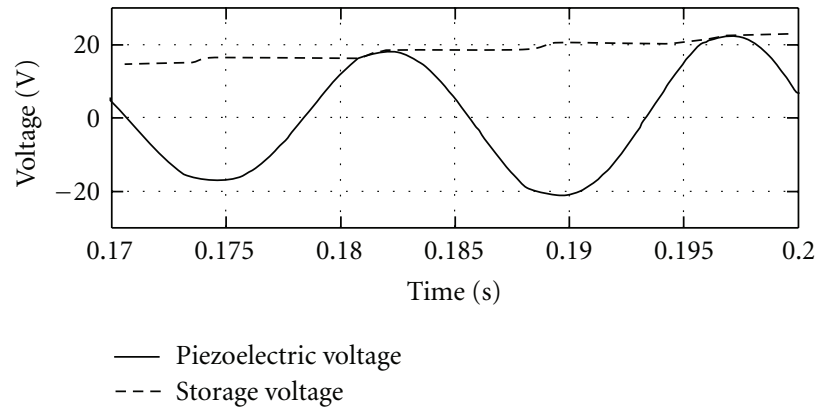

(a)

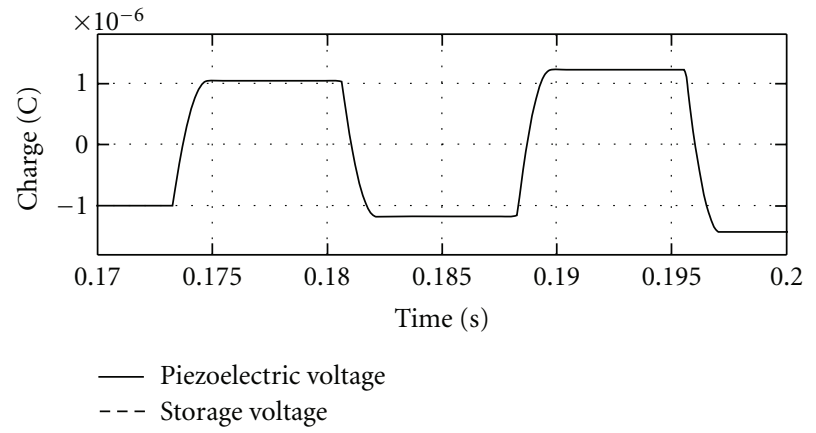

(b)

FIGURE 9: Magnified view of timeline of the tail wing with energy-harvesting (Circuit I). 


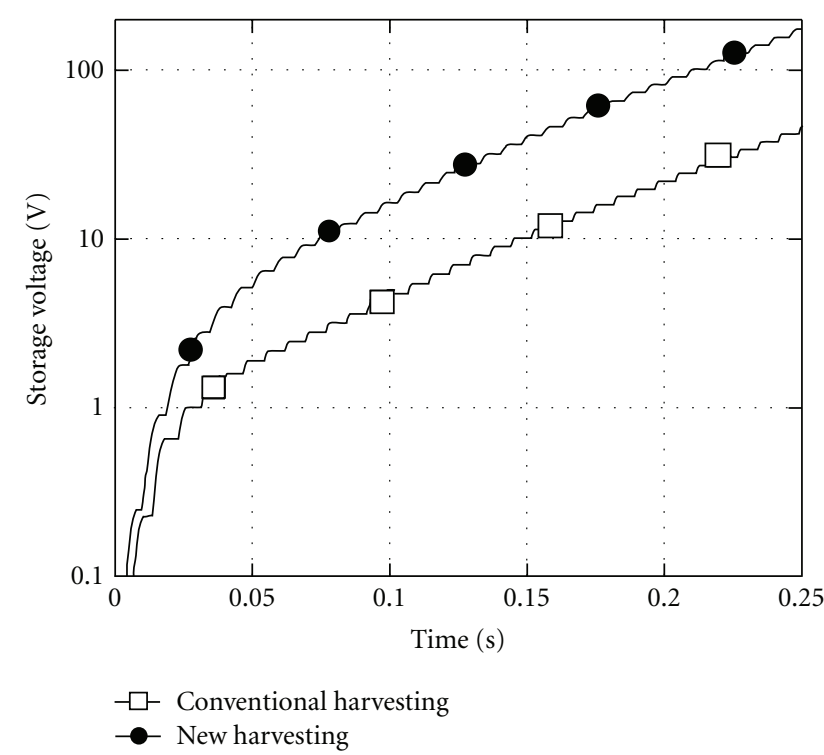

FIGURE 10: Comparison of stored voltages in the harvesting systems with conventional and new methods $(\kappa=1.0)$.

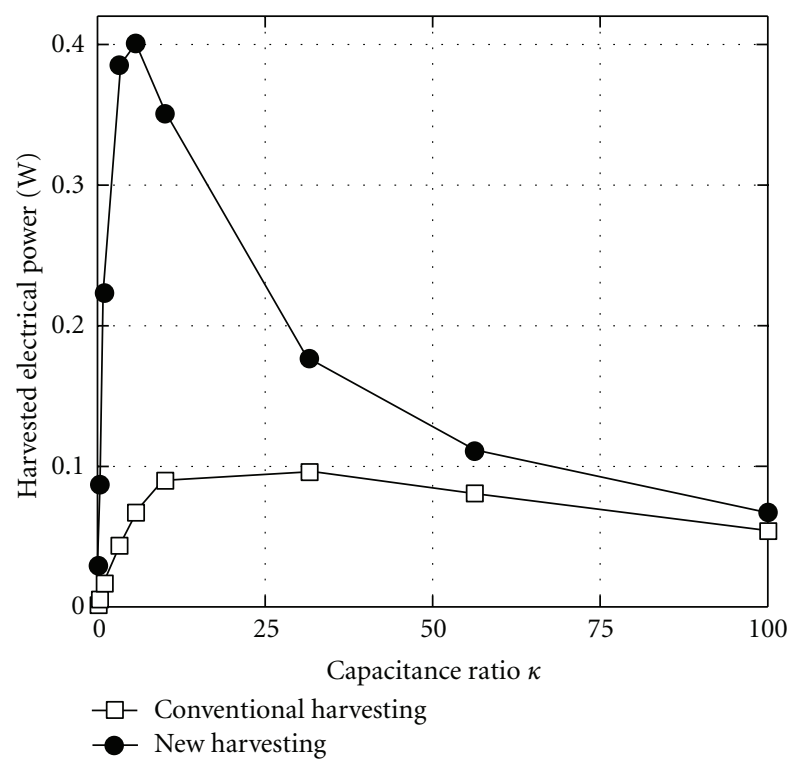

Figure 11: Harvested electrical power as a function of capacitance ratio $\kappa$ with conventional and new methods.

Secondly, although linear analysis indicates that flutter can often be expected to increase without limitation, nonlinear analysis of fluttering structures and aerodynamic theory suggest that the flutter phenomenon can lapse into limit cycle oscillation (LCO) [27-29]. Accordingly, if devices designed to work with intentional flutter are well built, flutter magnitude may not increase to infinity and cause critical failure. From this viewpoint, despite the conventional view of flutter as a destructive phenomenon, energy-harvesting based on the flutter phenomenon is quite a feasible concept with significant potential as a target for future research.
Lastly, we summarize the advantage and contribution of our harvesting method. These days, ecogeneration and ecofriendly inventions strongly attract more and more attention. Efficiency of energy harvesting is one of the most important issues for moving vehicles in aerospace engineering, such as airplanes and space vehicles. It is because oil fuel is quite costly and low-emission is requested by modern society. Our switching harvesting system through wing flutter can generate 10 times more electrical energy than a conventional harvesting system that is composed of a simple diode-bridge. This increase in harvested energy is a noteworthy number for the future development of power generation. This paper can provide a great potential of flutter harvesting and also change our notion against wing flutter from a harmful phenomenon to a profitable energy source.

\section{Conclusions}

We proposed a harvesting system that extracts electrical energy by effectively using wing flutter. This study employed the quasi-steady aerodynamic theory and the FEM for simulating the dynamics of a cantilevered-plate wing. The use of electric circuits and piezoelectric patches led to effective harvesting from supersonic flutter. We evaluated our switching approach using piezoelectric patches in supersonic flutter. Our proposed harvesting system via wing flutter can generate 10 times more electrical energy than conventional harvesting systems. We expect that our proposed technique will be applicable to various energy-harvesting systems, and we anticipate that it will be the basis for further studies in this field. More experimental validation is essential for assessing the harvesting performances, and an experiment is currently being carried out for this purpose. The effective utilization of flutter changes our perception of it-from being a destructive phenomenon to being useful in various applications.

\section{Nomenclature}

$a_{\infty}$ : $\quad$ Speed of sound in air

c: $\quad$ Young's modulus

$D_{z}, E_{z}: \quad z$-directional electric displacement and

electric field

f: $\quad$ External disturbance vector

F: $\quad$ Feedback matrix

$h_{x z}: \quad$ Piezoelectric coefficient

$n_{p}$ : Number of piezoelectric patches

$\mathbf{N}_{F}$ : $\quad$ Shape function of FEM element

$p: \quad \quad$ Pressure exerted on the plate

$p_{\infty}: \quad$ Air pressure

Q: $\quad$ Electric charge vector of piezoelectric patches

$T_{w}: \quad$ Kinetic energy of wing

$T_{p j}: \quad$ Kinetic energy of $j$ th piezoelectric patch

$u(x, y, t): z$-directional displacement of wing

$U: \quad$ Flight speed

$U_{w}$ : $\quad$ Strain energy of wing 
$U_{p j}$ : Mechanical and electrical energy of $j$ th piezoelectric patch

$V_{s}$ : Storage voltage in storage capacitor

V: Voltage vector of piezoelectric patches

x: Assembled displacement vector at FEM nodes

$\beta_{z z}^{S}: z$-directional dielectric coefficient at constant strain

$\mathcal{\varepsilon}, \sigma$ : Strain and stress vectors

$\eta$ : Modal displacement vector

$\rho, v$ : Density and Poisson's ratio

$\rho_{\infty}$ : Air density

$\zeta: \quad$ Modal damping ratio

$\gamma$ : $\quad$ Ratio of storage capacitance to piezoelectric capacitance.

\section{Superscript}

$D$ : Constant electric displacement

S: Constant strain

T: Transpose.

\section{Subscript}

$p:$ Piezoelectric patch

$p j$ : $j$ th piezoelectric patch

$w$ : Wing structure without piezoelectric patches.

\section{References}

[1] E. H. Dowell, "Nonlinear oscillations of a fluttering plate," AIAA Journal, vol. 4, no. 7, pp. 1267-1275, 1966.

[2] I. Lottati, "The role of structural and aerodynamic damping on the aeroelastic behavior of wings," Journal of Aircraft, vol. 23, no. 7, pp. 606-608, 1986.

[3] D. M. Tang and E. H. Dowell, "Experimental and theoretical study for nonlinear aeroelastic behavior of a flexible rotor blade," AIAA Journal, vol. 31, no. 6, pp. 1133-1142, 1993.

[4] Y. Odaka and H. Furuya, "Robust structural optimization of plate wing corresponding to bifurcation in higher mode flutter," Structural and Multidisciplinary Optimization, vol. 30, no. 6, pp. 437-446, 2005.

[5] S. H. Moon and J. S. Hwang, "Panel flutter suppression with an optimal controller based on the nonlinear model using piezoelectric materials," Composite Structures, vol. 68, no. 3, pp. 371-379, 2005.

[6] J. H. Han, J. Tani, and J. Qiu, "Active flutter suppression of a lifting surface using piezoelectric actuation and modern control theory," Journal of Sound and Vibration, vol. 291, no. 3-5, pp. 706-722, 2006.

[7] S. Raja, A. A. Pashilkar, R. Sreedeep, and J. V. Kamesh, "Flutter control of a composite plate with piezoelectric multilayered actuators," Aerospace Science and Technology, vol. 10, no. 5, pp. 435-441, 2006.

[8] A. Agneni, F. Mastroddi, and G. M. Polli, "Shunted piezoelectric patches in elastic and aeroelastic vibrations," Computers and Structures, vol. 81, no. 2, pp. 91-105, 2003.
[9] S. Beeby and N. White, Energy Harvesting for Autonomous Systems (Smart Materials, Structures, and Systems), Artech House, 2010.

[10] T. J. Kazmierski and S. Beeby, Energy Harvesting Systems: Principles, Modeling and Applications, Springer, 2010.

[11] P. J. Cornwell, J. Goethal, J. Kowko, and M. Damianakis, "Enhancing power harvesting using a tuned auxiliary structure," Journal of Intelligent Material Systems and Structures, vol. 16, no. 10 , pp. 825-834, 2005.

[12] W. P. Robbins, D. Morris, I. Marusic, and T. O. Novak, "Windgenerated electrical energy using flexible piezoelectric materials," in Proceedings of the ASME International Mechanical Engineering Congress and Exposition (IMECE '06), November 2006.

[13] S. D. Kwon, "A T-shaped piezoelectric cantilever for fluid energy harvesting," Applied Physics Letters, vol. 97, no. 16, Article ID 164102, 2010.

[14] K. Isogai, M. Yamasaki, and T. Asaoka, "Application of CFD to design study of flutter-power-generation," Special Publication of National Aerospace Laboratory, vol. 57, pp. 106-111, 2003 (Japanese).

[15] M. Bryant and E. Garcia, "Modeling and testing of a novel aeroelastic flutter energy harvester," Journal of Vibration and Acoustics, Transactions of the ASME, vol. 133, no. 1, Article ID 011010, 2011.

[16] C. De Marqui, A. Erturk, and D. J. Inman, "Piezoaeroelastic modeling and analysis of a generator wing with continuous and segmented electrodes," Journal of Intelligent Material Systems and Structures, vol. 21, no. 10, pp. 983-993, 2010.

[17] C. De Marqui Jr., W. G. R. Vieira, A. Erturk, and D. J. Inman, "Modeling and analysis of piezoelectric energy harvesting from aeroelastic vibrations using the doublet-lattice method," Journal of Vibration and Acoustics, Transactions of the ASME, vol. 133, no. 1, Article ID 011003, 2011.

[18] J. A. Dunnmon, S. C. Stanton, B. P. Mann, and E. H. Dowell, "Power extraction from aeroelastic limit cycle oscillations," Journal of Fluids and Structures, vol. 27, no. 8, pp. 1182-1198, 2011.

[19] V. C. Sousa, M. De Anicezio, C. De. Marqui, and A. Erturk, "Enhanced aeroelastic energy harvesting by exploiting combined nonlinearities: theory and experiment," Smart Materials and Structures, vol. 20, no. 9, article 094007, 2011.

[20] A. Erturk, W. G. R. Vieira, C. De Marqui, and D. J. Inman, "On the energy harvesting potential of piezoaeroelastic systems," Applied Physics Letters, vol. 96, no. 18, Article ID 184103, 2010.

[21] National Research Council Board, Sounding Rockets; Their Role in Space Research, General Books LLC, National Academy of Sciences, 2009.

[22] H. Ashley and G. Zartarian, "Piston theory-a new aerodynamic tool for the aeroelastician," Journal of the Aeronautical Sciences, vol. 23, pp. 1109-1118, 1956.

[23] B. Jaffe, W. R. Cook, and H. Jaffe, Piezoelectric Ceramics, Academic Press, London, UK, 1971.

[24] O. C. Zienkiewicz and R. L. Taylor, The Finite Element Method, Mcgraw-Hill, Berkshire, UK, 1985.

[25] J. Onoda, K. Makihara, and K. Minesugi, "Energy-recycling semi-active method for vibration suppression with piezoelectric transducers," AIAA Journal, vol. 41, no. 4, pp. 711-719, 2003.

[26] M. J. Balas, "Direct velocity feedback control of large space structures," Journal of Guidance, Control, and Dynamics, vol. 2, no. 3, pp. 252-253, 1979.

[27] J. E. Cooper and T. T. Noll, "Technical evaluation report on the 1995 specialists' meeting on advanced aeroservoelastic testing 
and data analysis," in Proceedings of the Conference Proceedings CP-566 (AGARD '95), 1995.

[28] M. J. Patil, D. H. Hodges, and C. E. S. Cesnik, "Nonlinear aeroelasticity and flight dynamics of high-altitude longendurance aircraft," in Proceedings of the 40th Structures, Structural Dynamics and Materials Conference, AIAA-1999-1470, Saint Louis, Mo, USA, 1999.

[29] J. P. Mayuresh, H. H. Dewey, and E. S. C. Carlos, "Limit cycle oscillations of a complete aircraft," in Proceedings of the 41st AIAA/ASME/ASCE/AHS/ASC Structures, Structural Dynamics, and Materials Conference, AIAA-2000-1395, Atlanta, Ga, USA, 2000. 

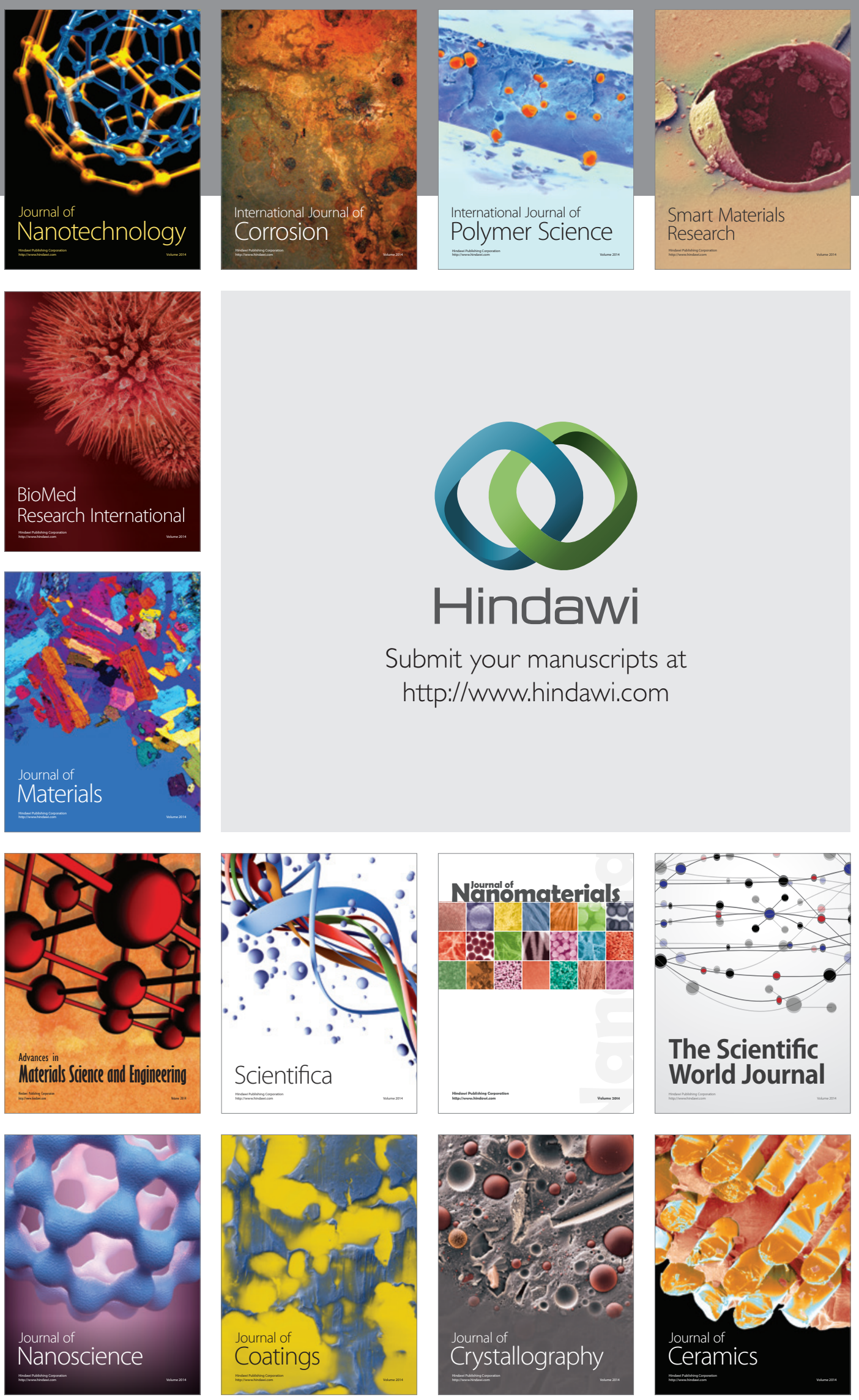

The Scientific World Journal

Submit your manuscripts at

http://www.hindawi.com

\section{World Journal}

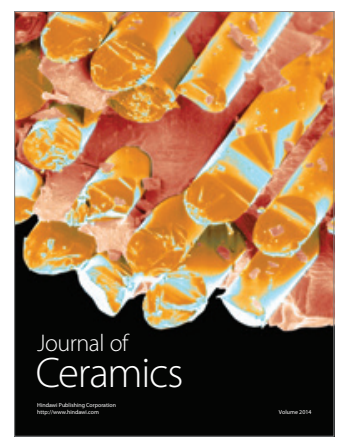

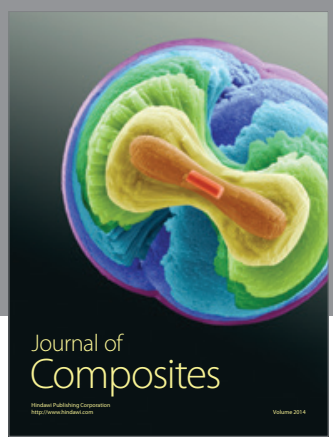
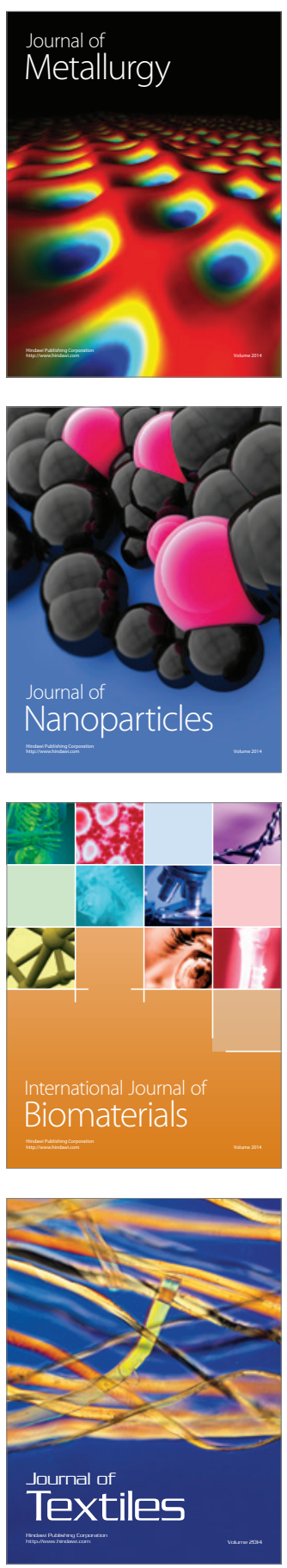